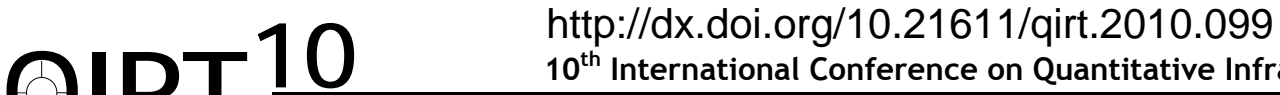 \\ $10^{\text {th }}$ International Conference on Quantitative InfraRed Thermography \\ July 27-30, 2010, Québec (Canada)
}

\section{DEFBETIR: Detection by infrared thermography of the heat signature of diseases and defects in concrete structures of civil engineering.}

by M. Nahant*, S. Paez** and J. Van Leeuwen***.

Construction Dept., Haute Ecole Robert Schuman, 2 chemin de Weyler, 6700 Arlon (Belgium).

*michael.nahant@hers.be - **samuel.paez@hers.be - ***jeremyvl@scarlet.be

\begin{abstract}
In this research, infrared thermography is used to detect the location of defects and damages in reinforced concrete structures. The research is mainly centred on the walls of wastewater treatment plants and existing bridge decks. The defects that need to be detected are rock pockets, delaminations and concrete disaggregation, but also all defects which have similar thermal properties. The aim of the project is to allow a defect mapping of the whole structure in a very short time, in order to repair it. Various heating techniques are being developed, as well as equipments able to do the inspections quickly and reliably on building sites.
\end{abstract}

1.

Introduction

Over the past few years, many techniques have been developed all over the world in order to evaluate in a nondestructive way the state of reinforced concrete structures, to detect the location and the extent of construction defects or damaged zones. Among these non-destructive techniques are the ultrasonic method, the ground penetrating radar, the impact-echo, the electric potential mapping, and the infrared thermography.

This project is led by two Belgian colleges, a construction company, and two public services. Its purpose is to use infrared thermography in order to detect defects or damages, located close to the surface, in two types of civil engineering buildings: wastewater treatment plants, and pre-stressed concrete bridge decks. These defects are principally rock pockets, but also cracks, cavities and concrete delaminations. Cracks and rock pockets affect both the water-tightness and the structural strength of concrete structures, whereas delaminations create a risk of flaking and falling of concrete fragments.

Infrared thermography has been chosen because it works without contact and it is swifter than the other techniques. It also allows the auscultation of a very large surface in a single time. The principle is the following : the concrete wall is heated either by the sun or by an infrared heater during a few minutes. Then some infrared images are taken with a thermographic camera. The defects (rock pockets principally, which are zones in the concrete where there is too little mortar within the gravel, or delaminations, which are cracks parallel to the surface) have a lower thermal conductivity than sound concrete. In defective areas, the heat stays near the surface whereas in sound areas it penetrates deeply into the concrete. Therefore the defective areas appear to be warmer on the infrared pictures.

2.

Finite elements pre-study

A tri-dimensional finite elements computer model has been developed in order to simulate the heat flow progression in a concrete material. It represents a cube of concrete of 10 centimetres of edge, which is part of a semi-infinite concrete wall. The cells of the model are cubes of $1 \mathrm{~cm}$ edge, so there are in all 1000 cells.

The model takes into account physical principles of conduction, convection and radiation. The attributes of each cell can be modified, so it is possible to put virtual defects anywhere in the model. The aim of this program is to simulate various configurations, like different defect depths, heating power or heating time, to check the value of the superficial thermal contrast.

The program computes the surface temperature evolution in front of sound concrete and in front of an internal defect. It gives as well the thermal contrast, which is the difference of the latter two. 


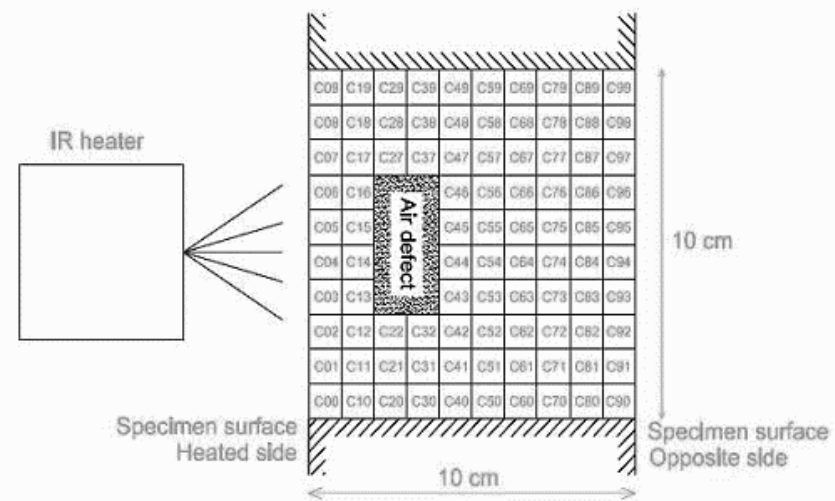

Fig. 1. Cross section of the model, showing a defect constituted of air.

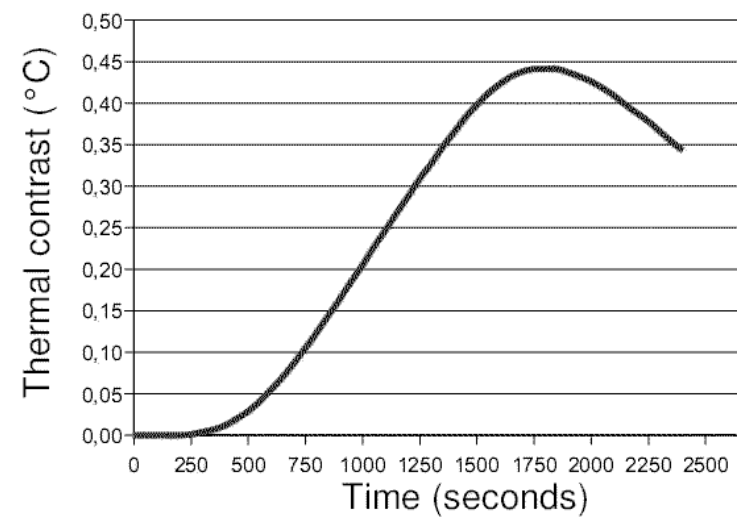

Fig. 2. Evolution of thermal contrast in front of a defect (in ${ }^{\circ} \mathrm{C}$ ) versus time (in seconds)

The information which can be drawn from this analysis is :

- Maximal thermal contrast is directly proportional to heating power,

- Maximal thermal contrast is also directly proportional to heating duration,

- $\quad$ Time of apparition of maximal thermal contrast is nearly independent of heating power, and doesn't vary very much with heating duration.

- $\quad$ Steel reinforcing bars have nearly no impact on the detection of defects,

- $\quad$ The maximal depth allowing the detection of defects is about $5 \mathrm{~cm}$,

- $\quad$ For a rock-pocket-like defect, at $4 \mathrm{~cm}$ depth, the maximal thermal contrast is about $15 \%$ of the temperature elevation of the surface of sound concrete at the same time.

- The effect of the wind is very important : a $20 \mathrm{~km} / \mathrm{h}$ wind divides by two the thermal contrast of a subsurface defect.

3.

Laboratory tests

Various concrete slabs have been made in order to simulate the defects that need to be detected : cracks, rock pockets and delaminations. Those tests have also been useful in validating the results provided by the finite elements model.

\subsection{Detection of cracks}

Some concrete elements have been broken and then re-assembled, in order to try to discover if infrared thermography is appropriate to detect cracks. The slabs were heated in a single point with a hot air heater. Tests have shown that the cracks slow the heat flow going from the heated point to the surroundings, showing the crack lines on the thermographs.
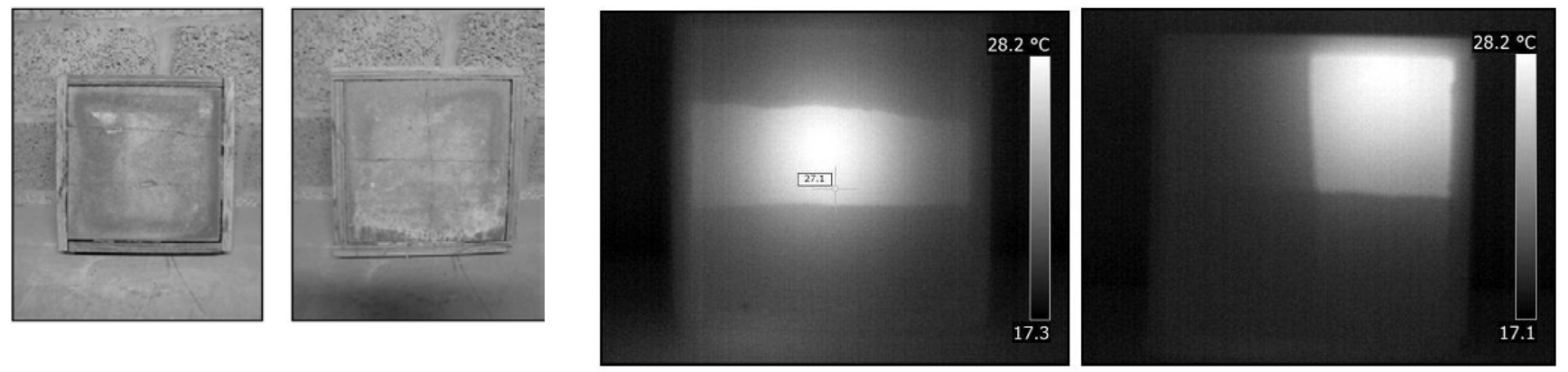

Fig. 3. Concrete slabs used for studying the detectability of cracks

Fig. 4. Infrared pictures of the slabs after local heating 


\subsection{Detection of rock pockets}

\subsubsection{Active thermography}

Some concrete slabs, containing gravel packets made to simulate rock pockets, have been realized. They have been studied using two different heating methods : static and moving. The heating, performed with an infrared heater, is not uniform enough with the static configuration, which adds an important difficulty to the detection of internal defects.
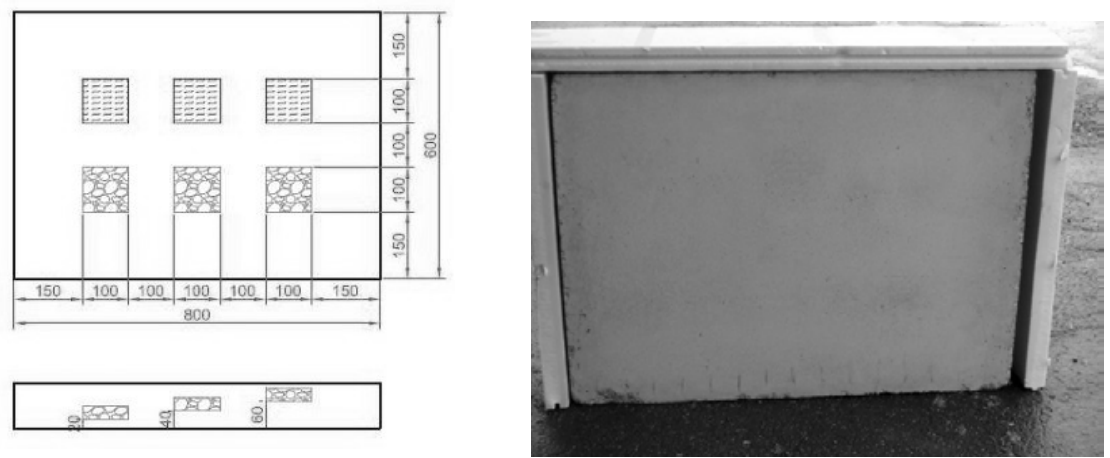

Fig. 5. Layout and picture of the concrete slab, containing polystyrene blocks (on top) and gravel packets (at the bottom)
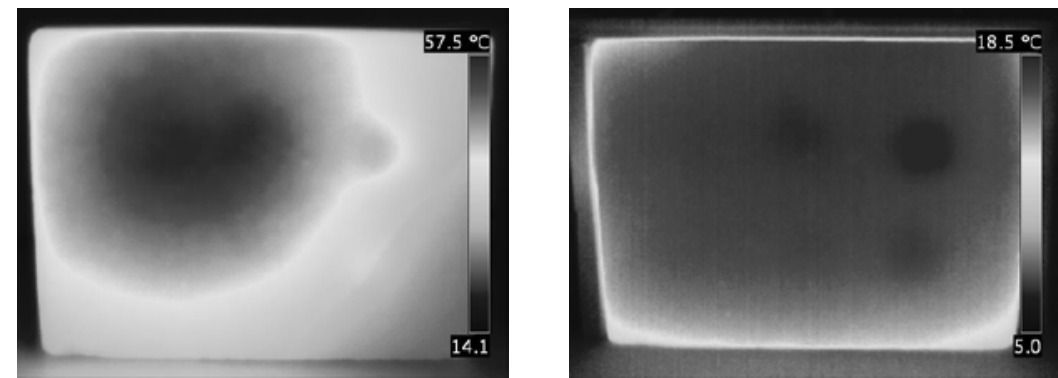

Fig. 6. Infrared pictures of the same slab. Comparison of both heating techniques : static heating (on the left) and moving heating (on the right). The second one gives a better uniformity and allows a better defect detection.

Consequently, it appears that the heating must be as even as possible to make reliable measures. For this purpose, moving is better than static heating. Moreover, when the test is carried out outside during a cold and windy day, it is much harder to detect the defects, due to the influence of wind and edge effects. It is thus better to protect the studied surface from the influence of the wind.

\subsubsection{Passive thermography}

The slab on the next thermogram has been exposed to sunlight for two hours, around noon time, a very sunny September day. On the infrared picture, the near-surface defects appear very clearly. Passive thermography can be used to locate rock pockets in concrete structures.

The main advantage of passive thermography is that it is both a cheap and an environment-friendly technique which provides a perfectly even heating. Disadvantages are the relatively low available heating power and the dependance to weather conditions, to surface orientation, and to the colour of the concrete, as the sun mainly heats in visible light.

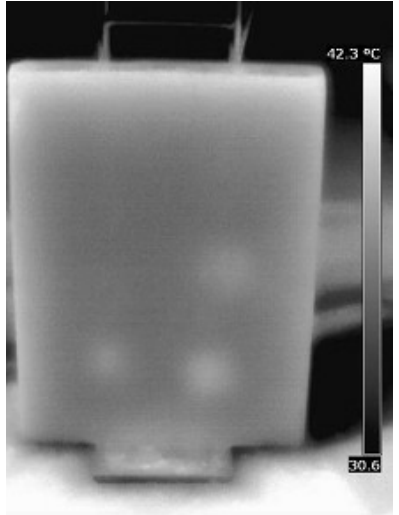

Fig. 7. Infrared picture of the slab : passive thermography (the only source of heating is the sun) 


\subsection{Verification of accuracy of infrared thermography with other detection techniques}

A very large concrete slab, containing a rock-pocket defect, has been studied by infrared thermography and three other techniques (ultrasounds, ground penetrating radar and coring), to check if the four results are matching.

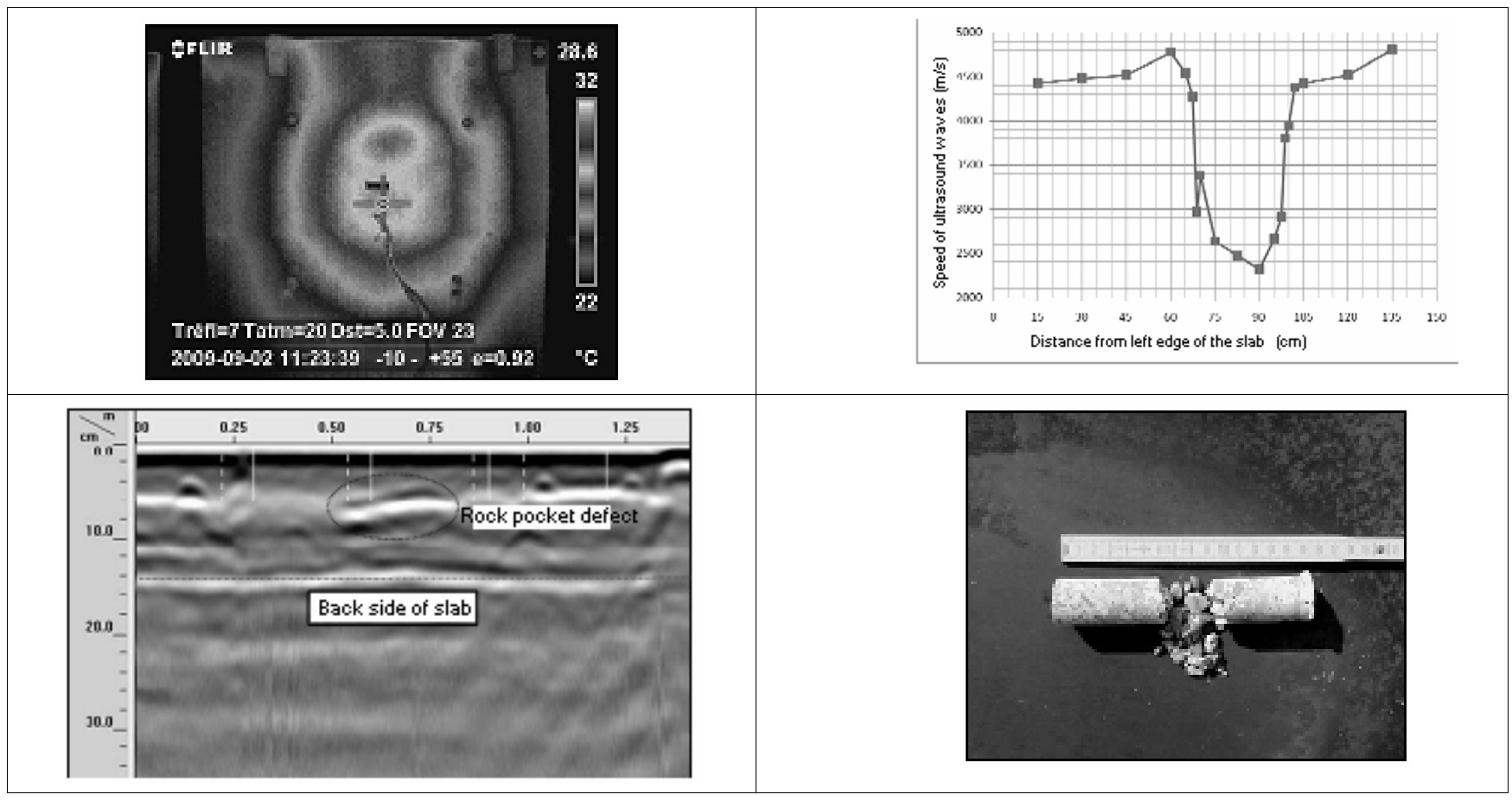

Fig. 8. Results of the four scannings. From top left to bottom right : active thermography, ultrasound, ground penetrating radar and coring.

The results of the four techniques match perfectly and locate the defect on the same spot. This shows that infrared thermography works as well as other proven techniques for detecting subsurface defects in concrete elements, and can be used on construction sites.

\subsection{Study of heating shape on a concrete wall}

On a real construction site, it is easier to perform a single passage of the heater rather than multiples ones. We tried both techniques on a concrete wall, to see if there are differences in heating uniformity.

The result of the study was that the technique of multiple passages (where the heater goes repeatedly past the tested zone) gives a better heating uniformity. It should be preferred whenever it is possible.

\subsection{Application of image processing techniques for detecting defects in concrete structures}

The principal problem involved in the detection of defects in concrete structures by active thermography is the system of heating. To obtain a good quality of detection, the thermal stimulation must be most uniform possible.

To be less dependent on this constraint, two temporal image processing techniques have been studied : the logarithmic derivative method, and pulsed phase thermography. Both of these methods require a great number of infrared pictures, taken at a regular time span, e.g. one per minute.

The main advantages of logarithmic derivative method and pulsed phase thermography are a better detectability of defects and a much lower sensitivity to non-uniformities of heating, irregularities and differences in surface colours. The challenge is the applicability of these methods under building site conditions to inspect concrete walls of wastewater treatment plants or bridge beams and decks. 


\subsubsection{Logarithmic derivative method}

This technique, developed recently by Shepard's group [4], consists in smoothing the surface temperatures cooling curve of each pixel of the image. Then the first and second derivatives are calculated, and images are reconstructed from those, identifying defects in a better way. The pictures below show the usefulness of this technique to decrease the importance of non-homogeneity of the heating.
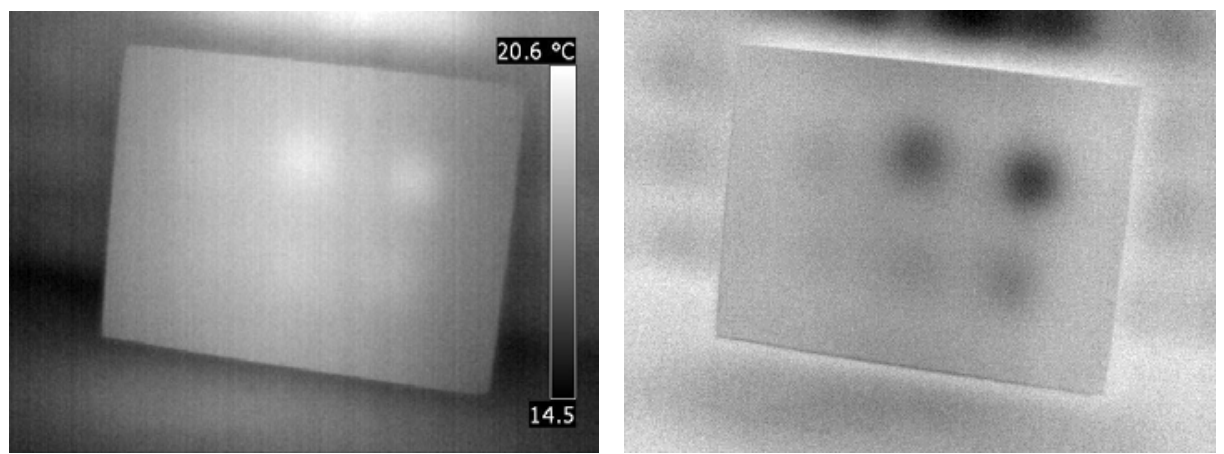

Fig. 9. Derivative method : the defects appear better on the derivative image (on the right) than on the original thermal image (on the left)

\subsubsection{Pulsed Phase Thermography}

Until now, pulsed phase thermography (PPT) was the subject of some studies led by people like Dr. Xavier Maldague [1] or Dr. Clémente Ibarra-Castanedo [3]. Their researches have developed the PPT for about fifteen years. However, these studies were confined at pointed industrial fields, like aeronautic or the aerospace. Moreover, these studies mainly concerned inspection of homogeneous material with a high thermal conductivity like aluminium or Plexiglas.

The application of PPT in the field of civil engineering for the study of concrete structures is relatively recent. Some studies were already realized on the PPT. One can mention those realized by the Federal Institute for Materials Research of Berlin (BAM) or by the Laboratoire d'Artois Mécanique Thermique Instrumentation (LAMTI).

Contrary to the previous studies performed on homogeneous materials with a high thermal conductivity, the application of this technique to a material like concrete is more delicate. Indeed, the thermal characteristics of concrete are radically different : the thermal conductivity of concrete is low and the studied thicknesses are much larger. These specificities imply that the scale of time of the analysis is strongly lengthened. The duration of the heating impulse of the former studies was a few milliseconds while, in the case of the concrete, the scale of the minutes is necessary. This lengthening applies, of course, with the phase of cooling which goes up from a few minutes to several hours. Moreover, the structure of the concrete is made up of a mixture of gravel, sand and cement. It is not homogeneous.

The defects to detect are rock pockets, which are zones where some mortar is missing between the aggregates. A poor concrete making, a bad implementation or an insufficient vibration during concreting are generally at their origin. They constitute weak points in the water-tightness and in the stability of the concrete walls. They present fuzzy contours and can take various forms. The rock pockets are particularly dangerous when they are near the steel reinforcements i.e. when they range roughly between 4 and 5 centimetres of surface. In the situation where water or other corrosive agents reaches these defects, a phenomenon of corrosion of steel bars may appear, with consequence of lack of resistance. The aim of the present research is the detection up to $6 \mathrm{~cm}$ depth from the surface.

First results are obtained by numerical simulation. After a heating phase the PPT is applied to the cooling phase. It appears that the phase images strongly reduce the non-uniformity of the heating, allowing a much better detectability. On the other hand the amplitude images do not improve the detectability. 

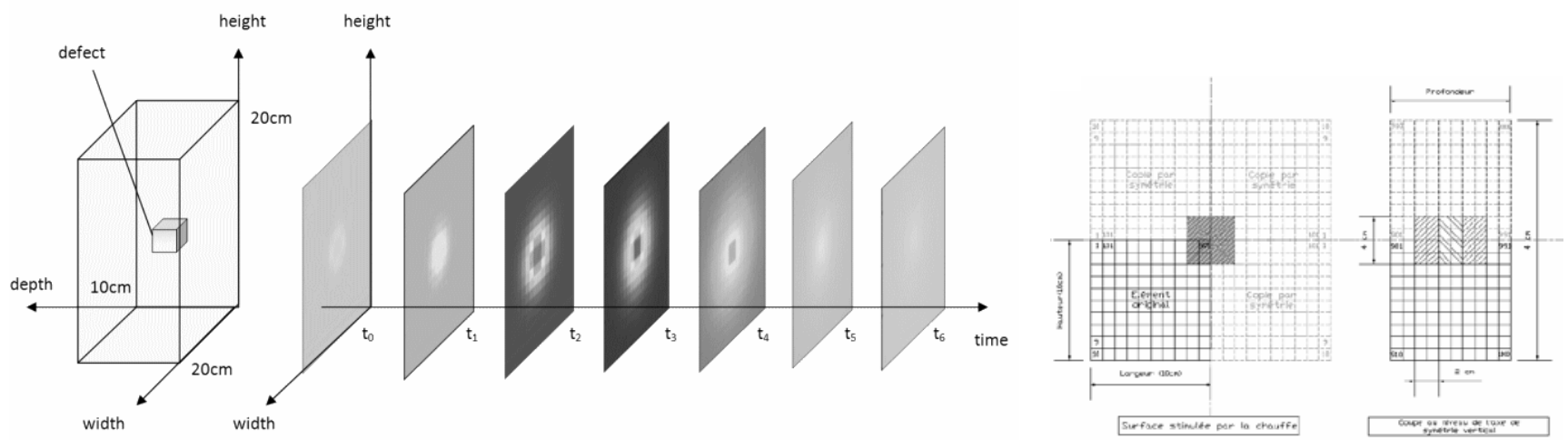

Fig 10 : Evolution of the surface temperature of a concrete element where a defect is inserted. (This defect consists of $50 \%$ of air and $50 \%$ of concrete)

Further PPT results are obtained on a test slab (fig 5) with defects at 2, 4 and $6 \mathrm{~cm}$ depth from the surface. The heating is artificial and uniform (fig $11 \mathrm{a}, \mathrm{b}, \mathrm{c}$ ) or non-uniform (fig $11 \mathrm{~d}, \mathrm{e}, \mathrm{f}$ ). The slab is heated during twenty minutes, then one infrared picture is taken per minute during a cooling time of 90 minutes. Like with the numerical simulations, best results are obtained with phase images which reveal all the defects in both conditions of heating.

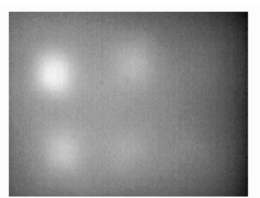

(a)

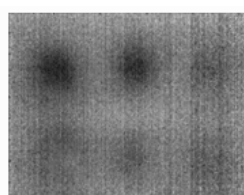

(b)

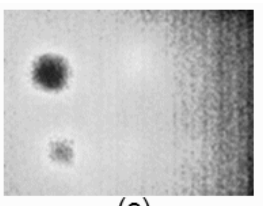

(c)

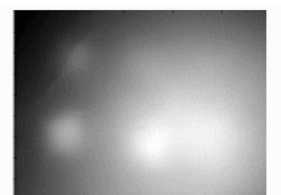

(d)

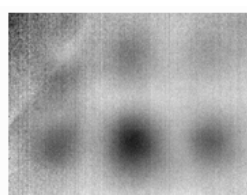

(e)

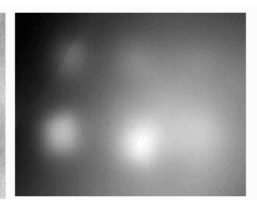

(f)

Fig. 11: (a), (d): infrared pictures ; (b), (e): phase images at 3,7 10 $\mathrm{Hz}$; (c), (f): amplitude images at $3,710^{-4} \mathrm{~Hz}$

Outdoor tests are performed on the same slab, still with artificial heating but under real climatic conditions disturbing the detection : the variations of sun power and wind speed during the experiment create disturbances in the cooling curve. Moreover, the slab has zones where the surface colours are different. The darker zones receive more solar energy, they become thus hotter and the infrared pictures are strongly disturbed by this phenomenon. Information on phase images is thus reduced. The defects are clearly visible on the first image of phase (fig $12 \mathrm{c}$ ). On the following images of phase, they are no more detectable (fig $12 \mathrm{~d}$ ). To improve the images of phase, the cooling curve of each pixel is smoothed. This operation allows to obtain a better detectability on several images of phase (fig $12(\mathrm{e})$, (f)).

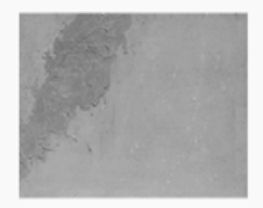

(a)

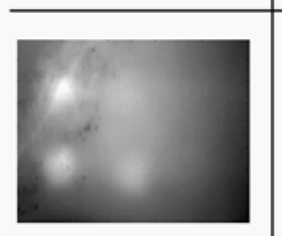

(b)

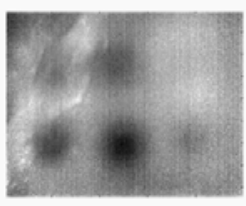

(c)

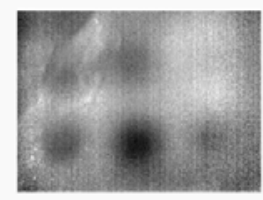

(e)

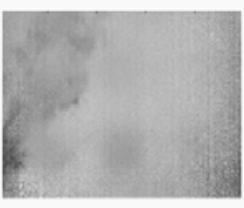

(d)

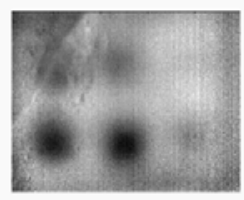

(f)

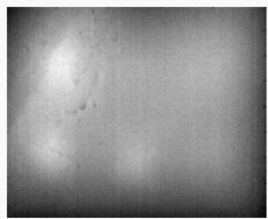

(a)

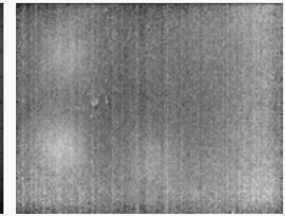

(b)

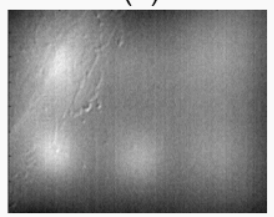

(e)

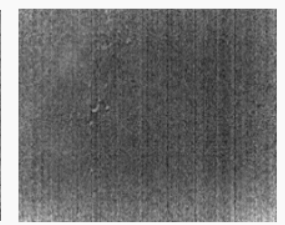

(c)

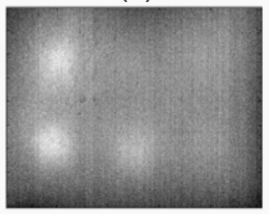

(f)

Fig. 12: Artificial heating. (a): photograph of the test slab; (b): infrared picture; (c) and (d): phase images at 1,85 10 $\mathrm{Hz}$ and 3,7 10 $\mathrm{Hz}$; (e) and (f): phase images at 1,85 $10^{-4}$ $\mathrm{Hz}$ and $3,710^{-4} \mathrm{~Hz}$ with smoothing. 
On fig. 13 the PPT is tested with a solar stimulation. The cooling phase is done by protecting the slab from the solar rays. The low power of heating produces a small fall of temperature. This reason explains why the phase images (fig $13 \mathrm{~b}, \mathrm{c}$ ) reveal the defects slightly. On the other side, the amplitude images (fig $13 \mathrm{e}, \mathrm{f}$ ) improve the detection of the defects and attenuate the influence of difference in surface colour when heating is realized by the sun.

4.

\section{On-site auscultations}

\subsection{Bridge of Moyen}

This reinforced concrete bridge is known for containing many rock-pocket defects. A passive thermography survey has been carried out to locate them. Here are the most significant pictures where subsurface defects are circled.
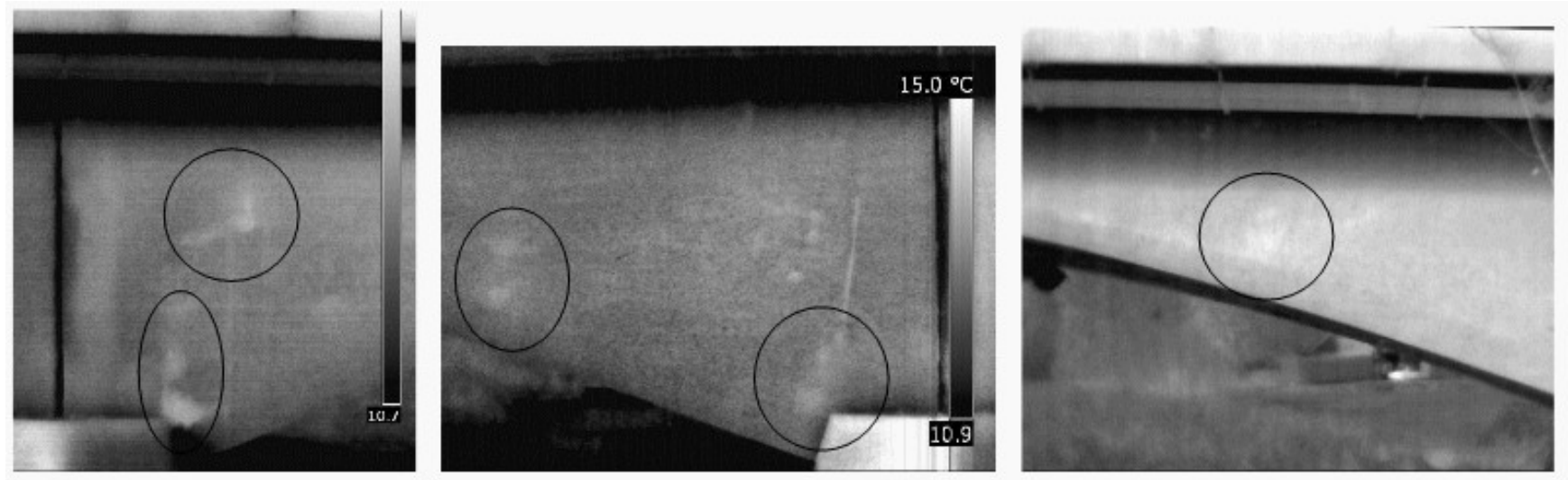

Fig. 14. Infrared pictures taken at the bridge of Moyen with passive thermography. Rock pockets appear as warmer zones.

\subsection{Wastewater treatment plants (WWTP)}

A goal of the project is to allow defect mapping of a whole biological pool of a wastewater treatment plant in only one week, with a fast and industrial process. If the defects are clearly located, they can be repaired before the starting up of the WWTP, and the need for expensive water-proofness tests is highly reduced.

Special constraints for the present project are the dimensions of the purification pools : the surfaces that need to be analyzed by thermography vary from a hundred square meters to several thousands. The walls of those tanks are made of high quality reinforced concrete, and they are sometimes thicker than thirty centimetres.

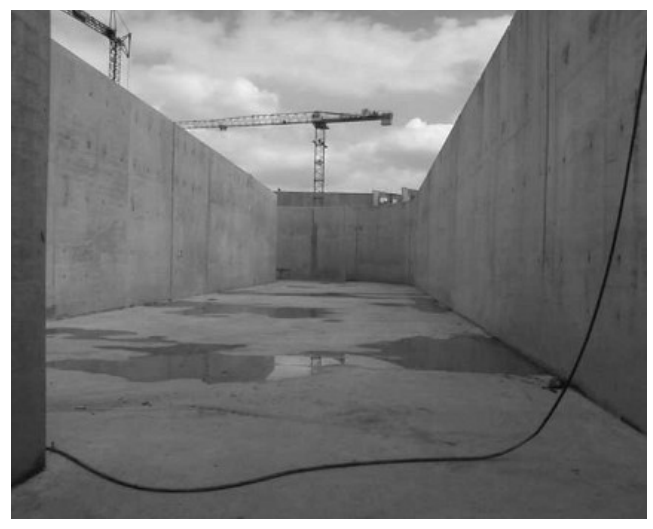

Fig. 15. The biological tank of a wastewater treatment plant 
An active thermography auscultation has been carried out, and an alleged defect appeared on the pictures.
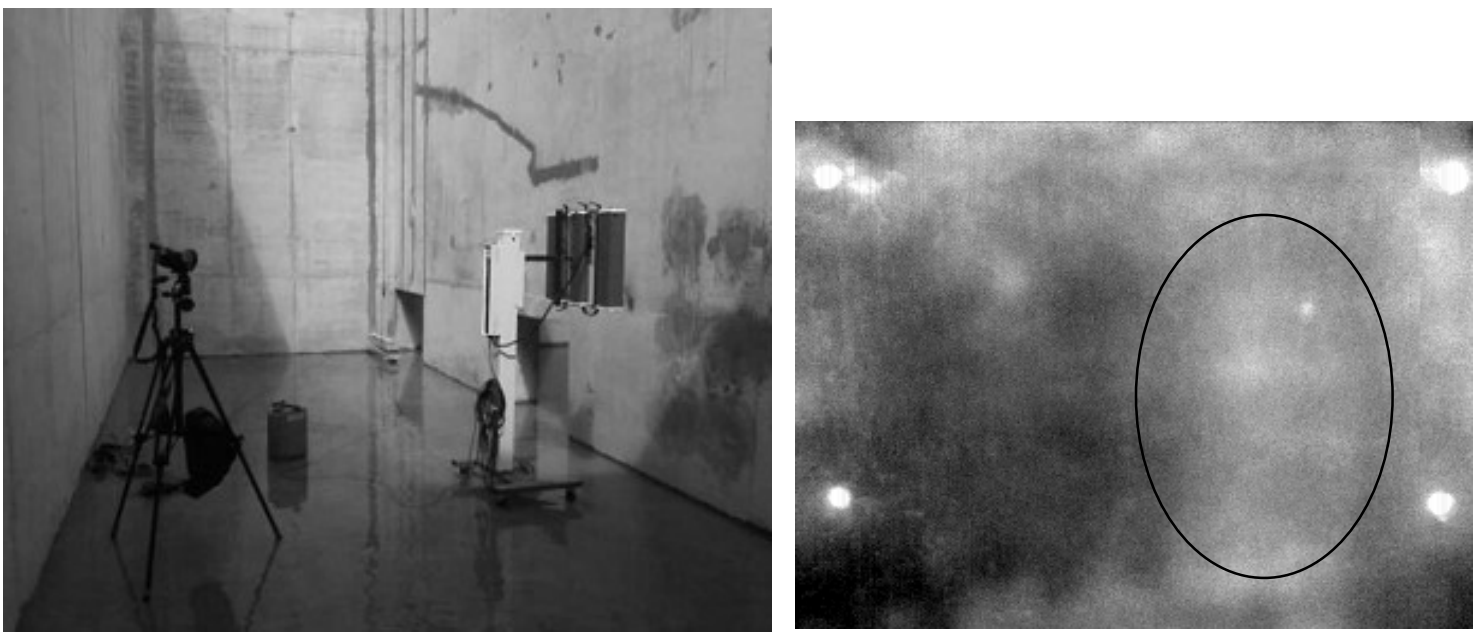

Fig. 16. A warmer zone appears on the thermographic picture, probably showing up the presence of a internal defect.

5.

\section{Main innovations}

Three main innovations in the present project are :

- $\quad$ The use of infrared thermography to detect mainly rock pockets. These are more difficult to detect than other defects, like cavities or air bubbles under sealing capes of bridges. Indeed, the difference of thermal conductivities are higher between air and concrete than between rock pocket and concrete.

- $\quad$ The considerable dimensions and the significant curve of the surfaces that have to be studied. The concrete structures presently investigated by thermography are considerably smaller. Here the analysis must be performed on an industrial and large-scale way, while remaining very fast.

The development of alternative heating techniques dealing with special aspects of civil engineering structures: surface emissivity variations, accessibility of the studied area, lack of uniformity of the heating, intrusive reflections on the concrete surface, influence of the wind, etc.

\section{REFERENCES}

[1] Maldague X., Theory and Practice of Infrared Technology for Nondestructive Testing, Wiley, 2001.

[2] Sham Fung Chu J., Studies of using Infrared Flash Thermography (FT) for detection of surface cracks, subsurface defects and water-paths in building concrete structures, City University of Hong-Kong, 2008.

[3] Ibarra-Castanedo C., Quantitative subsurface defect evaluation by Pulsed Phase Thermography : Depth retrieval with the phase, Université Laval, Québec, 2005.

[4] Shepard S. M., Lhota J. R., Rubadeux B. A., Wang D. and Ahmed T., Reconstruction and Enhancement of Active Thermographic Image Sequences, Optical Engineering N. 42, 2003. 